University of Warwick institutional repository

This paper is made available online in accordance with

publisher policies. Please scroll down to view the document

itself. Please refer to the repository record for this item and our

policy information available from the repository home page for further information.

To see the final version of this paper please visit the publisher's website. Access to the published version may require a subscription.

Author(s): F M Poli, S E Sharapov, S C Chapman

Article Title: Study of the spectral properties of ELM precursors by means of wavelets

Year of publication: 2008

Link to published version:

http://dx.doi.org/10.1088/0741-3335/50/9/095009

Publisher statement: None 


\title{
Study of the spectral properties of ELM precursors by means of wavelets
}

\author{
F. M. Poli \\ Department of Physics, University of Warwick, Coventry, CV4 7AL, UK. \\ E-mail: f.m.poli@warwick.ac.uk \\ S. E. Sharapov \\ Euratom/UKAEA Fusion Association, Culham Science Centre, Abingdon OX14 \\ 3DB, UK
}

\section{S. C. Chapman}

Department of Physics, University of Warwick, Coventry, CV4 7AL, UK.

\section{JET-EFDA contributors}

\begin{abstract}
The high confinement regime (H-mode) in tokamaks is accompanied by the occurrence of bursts of MHD activity at the plasma edge, so-called edge localized modes (ELMs), lasting less than $1 \mathrm{~ms}$. These modes are often preceded by coherent oscillations in the magnetic field, the ELM precursors, whose mode numbers along the toroidal and the poloidal direction can be measured from the phase shift between Mirnov pickup coils. When the ELM precursors have a lifetime shorter than a few ms, their toroidal mode number and their nonlinear evolution before the ELM crash cannot be studied reliably with standard techniques based on Fourier analysis, since averaging in time is implicit in the computation of the Fourier coefficients. The present work demonstrates significant advantages in studying spectral features of the shortlived ELM precursors by using Morlet wavelets. It is shown that the wavelet analysis is suitable for the identification of the toroidal mode numbers of ELM precursors with the shortest lifetime, as well as for studying their nonlinear evolution with a time resolution comparable to the acquisition rate of the Mirnov coils.
\end{abstract}

PACS numbers: $52.25,52.35,52.55$

$\ddagger$ See the Appendix of M. L. Watkins et al., Fusion Energy 2006 (Proc. 21st Int. Conf. Chengdu, 2006) IAEA, (2006) 


\section{Introduction}

The high confinement regime (H-mode) in tokamaks is often accompanied by bursts of MHD activity, associated with an increase in the $\mathrm{D}_{\alpha}$ emission at the edge [1]. These instabilities, occurring at the edge and therefore known as edge localized modes (ELMs) are the cause of particle and energy losses that may result in a reduction of the energy confinement time as large as 20\% [1]. ELMs are commonly classified on the basis of the occurrence of magnetic precursors and on the dependence of the ELM repetition frequency on the energy flux through the separatrix [1][2][3][4]. According to this criteria one can identify two main classes of ELMs, type-I and type-III, depending on whether the repetition frequency increases or decreases with the energy flux.

On the JET tokamak, both type-I and type-III ELMs are MHD events lasting between 0.3 and $1 \mathrm{~ms}$; magnetic precursors of type-I ELMs have been detected lasting from a few ms to hundredths of ms. These precursors were studied by Perez et al [5], although precursors with lifetime shorter than a few milliseconds were excluded from that analysis due to the intrinsic difficulty of assessing them with standard Fourier techniques. This work focuses on the study of the spectral properties of these short-lived perturbations by using Morlet wavelets, i.e. sinusoidal modulated functions, instead of Fourier eigenmodes, for the first time. A number of plasma discharges in H-mode have been analyzed with wavelets, with both type-I and type-III ELMs. However, for the purposes of this paper, aimed at introducing and describing the wavelet analysis applied to ELMs and to ELM precursors, we focus on one typical example, a H-mode plasma discharge with type-I ELM precursors and show the advantages of wavelet analysis with an example for type-III ELMs. The method and the basic concepts of wavelet analysis are introduced in section 2. The results and possible pitfalls are discussed in section 3, illustrating as an example the linear and nonlinear spectral features of a short-lived type-I ELM precursor. Finally, future applications of wavelet analysis for studying the toroidal mode number and the nonlinear evolution of ELMs are discussed in section 4.

\section{Method}

Fourier analysis has traditionally been the starting point to analyse stationary fluctuations in a plasma, under the hypothesis that the signal can be regarded as a linear superposition of independent, monochromatic, sinusoidal waves. If the fluctuations are not stationary, the variations of the wave field amplitude and phase over short time scales are not captured in the Fourier spectrum due to time averaging implicit in the Fourier transform. In this case a representation of the plasma wave fields in terms of wavelets is more appropriate. We first briefly review relevant concepts in wavelet analysis, and refer the reader to Mallat [6] for a complete and extensive discussion of wavelets and time-frequency analysis. The Morlet wavelet is the natural choice when the focus of the analysis is spectral features, as it essentially represents Fourier eigenmodes 

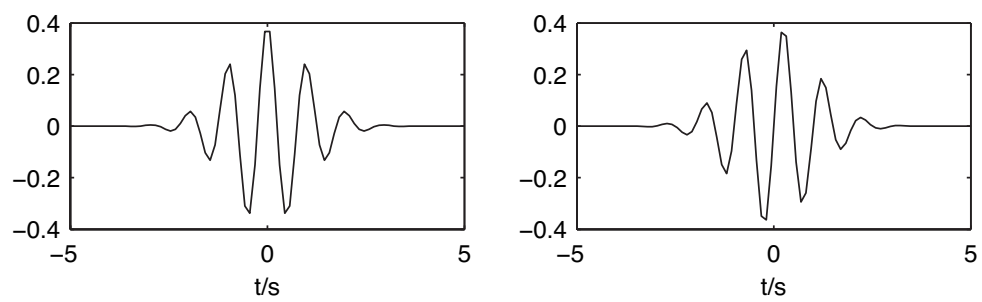

Figure 1. Real (left) and imaginary (right) part of the Morlet wavelet, equation (1), for $\omega_{0}=2 \pi$ and $\sigma=1$.

whose energy is localized in time. It is defined as:

$$
\psi(t)=\pi^{-1 / 4} e^{-t^{2} / 2 \sigma^{2}} e^{\imath \omega_{0} t}
$$

and its real and imaginary part are illustrated in figure 1 in the case of $\sigma=1$ and $\omega_{0}=2 \pi$. A family of wavelets can be constructed from (1) by scaling and shifting $\psi(t)$ :

$$
\psi_{s \tau}(t)=\frac{1}{\sqrt{s}} \psi\left(\frac{t-\tau}{s}\right)=\left(\pi s^{2}\right)^{-1 / 4} e^{-\frac{(t-\tau)^{2}}{2 s^{2}}} e^{2 \pi \imath \frac{t-\tau}{s}},
$$

where the factor $(s)^{-1 / 2}$ has been introduced for normalization and we have set $\sigma=1$ and $\omega_{0}=2 \pi$. There is thus a clear point of contact with Fourier eigenmodes, of frequency $\omega$, that are time localized over $s$.

The continuous wavelet transform (CWT) of a discrete time series $x_{n}$, sampled at the rate $\delta t$, is defined as the convolution product of $x_{n}$ with $\psi_{s \tau}(t)$ :

$$
W_{m}(s)=\sum_{n=0}^{N-1} x_{n} \psi^{*}\left(\frac{n-m}{s} \delta t\right)
$$

Apart from normalization factors, the only difference between (3) and the windowed Fourier transform is that the windowing is intrinsic in the wavelet transform and it depends on scale.

Equation (3) is computationally highly inefficient, since it involves $N^{2}$ operations. Using the property that the Fourier transform of a convolution product is the product of the Fourier transforms, algorithms based on the Fast Fourier Transform can be used to calculate the CWT, with considerable efficiency gain. The wavelet transform is computed at scales $s=s_{0} a^{j}$, where $s_{0}$ is the minimum available scale and, for each value of $j, a=2^{-\nu}$, where $\nu$ is an integer number that provides a refining of scales in each octave $\left(2^{j}, 2^{j+1}\right][6]$.

Spectral quantities, such as the linear dispersion relation, are defined for wavelets in the same way as for Fourier transforms. From two time series measured at toroidal locations $\phi_{1}$ and $\phi_{2}$ respectively, the toroidal mode number at each time $t_{m}=m \delta t$ can be extracted from the relative phase shift as:

$$
n_{m}(\phi, s)=\frac{1}{\Delta \phi} \arg \left[W_{m}^{*}\left(\phi_{1}, s\right) W_{m}\left(\phi_{2}, s\right)\right]
$$

where $W_{m}^{*}\left(\phi_{1}, s\right) W_{m}\left(\phi_{2}, s\right)$ is the cross-power spectrum between the two signals. For the sake of simplicity, the explicit dependence on the position $\phi_{1,2}$ will be omitted in the 
rest of the paper.

In the same manner as with Fourier transforms, wavelets can be used to calculate higher order spectra, such as the bispectrum [7]. Wavelets can however quantify the degree of nonlinear coupling over time scales shorter than those accessible by Fourier analysis. The wavelet bispectrum between $t_{1}=m_{1} \delta t$ and $t_{2}=\left(m_{1}+M\right) \delta t$ is defined as:

$$
B\left(s_{1}, s_{2}\right)=\frac{1}{M} \sum_{m=m_{1}}^{m_{1}+M} W_{m}\left(s_{1}\right) W_{m}\left(s_{2}\right) W_{m}^{*}\left(s_{3}\right)
$$

where the scales $s_{1}, s_{2}$ and $s_{3}$ are such that the associated frequencies satisfy the resonance condition $\omega_{1}+\omega_{2}=\omega_{3}$. If nonlinear interactions are present in a time series, both their frequencies and their phases are correlated according to the resonance condition, so that the value of $\left|B\left(s_{1}, s_{2}\right)\right|$ converges to a finite value as $M$ becomes large. Equation (5) is valid provided that the degree of nonlinearity is weak and that fluctuating quantities can therefore be represented as (time localized) sinusoidal waves [7][8]. The value of $M$ should be taken as large as possible to guarantee convergence of the bispectrum [7]. Typically the average in (5) should be calculated over time windows much longer than the wave period, although the number of required samples may be reduced if the signal-to-noise ratio is high and/or if only a few coupled modes are present in the spectrum. The suitability of wavelets to calculate higher order spectra in order to isolate short-lived, coherent, nonlinear structures in intermittent plasma time series was discussed by van Milligen et al [9].

An estimate of the bispectrum, independent of the power spectrum of the interacting components, is provided by the bicoherence [8]:

$$
b^{2}\left(s_{1}, s_{2}\right)=\frac{\left|\sum_{m} W_{m}\left(s_{1}\right) W_{m}\left(s_{2}\right) W_{m}^{*}\left(s_{3}\right)\right|^{2}}{\sum_{m}\left|W_{m}\left(s_{1}\right) W_{m}\left(s_{2}\right)\right|^{2} \sum_{m}\left|W_{m}\left(s_{3}\right)\right|^{2}}
$$

Importantly, although algorithms based on the Fast Fourier Transform are more computationally efficient methods to calculate the CWT, equation (3) is more suitable for the computation of higher order spectra. When the time series is decomposed in terms of diadic bases, in fact, the logarithmic scales $\log _{2}(s)$ form a uniform grid with separation $\nu^{-1}$. On the other hand, due to the inverse proportionality between $\omega$ and $s$, the resulting frequency grid is not uniform. This reflects in the inability of satisfying the resonance condition among frequencies.

Let $\omega_{1}, \omega_{2}$ and $\omega_{3}$ be three spectral components that satisfy $\omega_{1}+\omega_{2}=\omega_{3}$. Using the relation between frequency and scale and after some manipulation, the resonance condition among frequencies corresponds to the following relation among scales:

$$
\log _{2} s=\log _{2} s_{1}+\log _{2} s_{2}-\log _{2}\left(s_{1}+s_{2}\right)
$$

Because of the last term on the right hand side, the term on the left hand side is not necessarily associated to any point in the computed grid of scales and thus $\omega_{3}$ does not match $2 \pi / s$. When calculating higher order spectra it is therefore preferable to fix the frequency grid first, then compute the corresponding scales and use equation (3) to compute the CWT. 

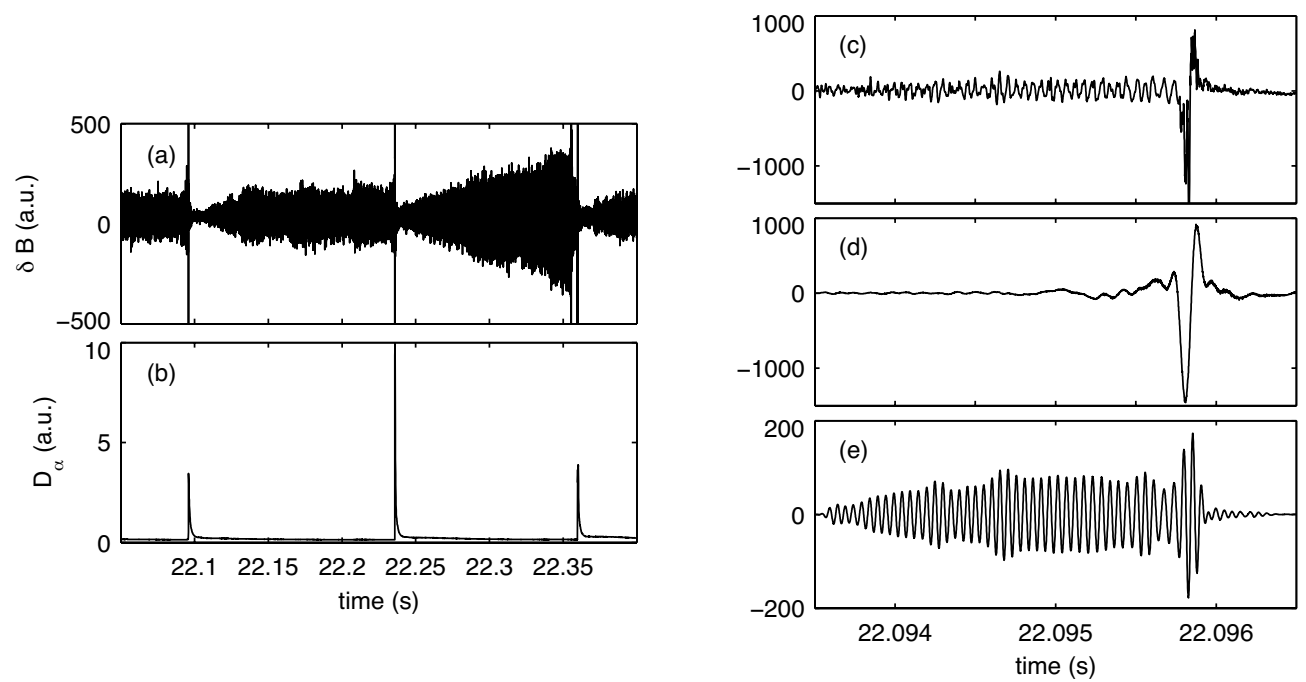

Figure 2. (a) Time trace of the magnetic fluctuations measured in JET pulse \#53062, with an outer edge Mirnov pick-up coil, positioned at $R=3.881 \mathrm{~m}, z=1.013 \mathrm{~m}$, sampled at $1 \mathrm{MHz}$, during $0.4 \mathrm{~s}$ of current flat-top discharge with constant NBI power of $13 \mathrm{MW}$. (b) Time trace of $\mathrm{D}_{\alpha}$ emission, measured in the outer divertor and sampled at $10 \mathrm{kHz}$. (c) Excerpt of magnetic perturbations, with zoom on the first ELM precursor. (d) Wavelet coefficients in the range of frequencies $[2,10] \mathrm{kHz}$. (e) Same as (d), but in range of frequencies $[18,20] \mathrm{kHz}$.

\section{Results}

\subsection{Type-I ELM precursors}

Precursors to the ELMs are observed on JET as magnetic perturbations in the signal measured by a set of calibrated edge Mirnov pickup coils [10]. We will now use the concepts introduced in the previous section to study the evolution in time of the spectral features of ELM precursors, such as the amplitude, the frequency and the toroidal mode number. We will use for the analysis the magnetic perturbations measured by two Mirnov coils, positioned at the same major radius, $R=3.881 \mathrm{~m}$, at $z_{1}=1.013 \mathrm{~m}$ and $z_{2}=1.005 \mathrm{~m}$ above the midplane, and with orientation $\theta=108.9$ degrees between the coil axis and the major radius. The two coils are separated by $\Delta \varphi=10.17$ degrees along the toroidal direction, allowing the measurement of toroidal mode numbers up to $|n|=17$. This subset of coils is sampled at $1 \mathrm{MHz}$, thus providing good resolution in time. Although the wavelet analysis has been applied to study a number of $\mathrm{H}$ mode plasma discharges on JET with type-I ELMs, we concentrate on one example - typical for most of the cases considered so far - to illustrate the technique. The chosen discharge pulse \#53062 is a typical ELMy plasma with type-I ELMs, in which precursors are detected. All the precursors observed in this discharge have similar frequency and toroidal mode number, but their lifetime can vary from less than $1 \mathrm{~ms}$ to hundredths of ms. In the case of the longer-lived precursors the results with wavelets can be directly compared with the Fourier analysis, thus providing a test of reliability 
for the wavelet analysis. The precursors with longer lifetime were analyzed in detail by Perez et al [5] in the framework of an extensive study of type-I ELM precursors. The analysis in that work was restricted to precursors with lifetime typically a few tens of milliseconds, the choice being dictated by the difficulty of discerning in the Fourier spectrogram precursors with lifetime shorter than a few ms. The time dependent Fourier spectra of the amplitude of magnetic perturbations and the associated toroidal mode number for this discharge are shown in figures 2-3 of [5] and therefore they are not reported here. Figure 2shows the time trace of magnetic perturbations measured in the time window between 22.0 and $22.4 \mathrm{~s}$. Three ELMs can be seen to occur in the time window of interest; they are associated with large spikes in the magnetic perturbations that correlate with the peaks detected in the time traces of the $\mathrm{D}_{\alpha}$ emission. In figure 3 we can see that significant spectral energy is associated with the ELMs and with coherent modes in the power spectrum, such as the ELM precursors at frequency $\sim 20$ $\mathrm{kHz}$ and the sawtooth precursor at frequency $\sim 15 \mathrm{kHz}$. The exact correspondence between frequencies and scales has been verified by computing the CWT of a cosine function with given frequency. For graphical representation purposes, for each scale $s$ the wavelet coefficients in figure 3 have been rescaled with respect to the difference between the maximum and the minimum value measured at that scale:

$$
\left|W_{m}^{\prime}(s)\right|=\frac{\left|W_{m}(s)\right|-\left|W_{m}(s)\right|_{\min }}{\left|W_{m}(s)\right|_{\max }-\left|W_{m}(s)\right|_{\min }} \times 256
$$

The final values of $\left|W_{m}^{\prime}(s)\right|$ are bounded between 0 and 256 .

Oscillations at constant frequency below $1 \mathrm{kHz}$, that is at $\log _{2}(s)>12$, are detected in the wavelet spectrum, they are most probably related to external power supplies on JET. One should therefore discard all wavelet coefficients corresponding to scales $s_{j}$ such that $\log _{2}(s)>12$. A broadening in time is observed in the computed wavelet scalogram for the largest scales, typically larger than $\log _{2}(s)>12$, in time windows centred on the ELMs. This feature, which is more evident for the second ELM in figure 3 , is observed also in other discharges and it appears to occur for large amplitude type-I ELMs, namely those associated with higher $\mathrm{D}_{\alpha}$ emission.

Figure 3 also shows a plot of the toroidal mode number calculated from the phase shift between the two Mirnov coils, using equation (4). From the figure we can see the following. First, for the precursor to the second ELM, which has longer lifetime, shown in figure 3-(c), the toroidal mode number calculated with the wavelet analysis is $n \sim 8$ and consistent with the results from Fourier analysis discussed in [5]. For the precursor to the first ELM, which has much shorter lifetime, shown in figure 3-(b), the toroidal mode number is also $n \sim 8$, as we would expect, since this is the value found for the longer lived precursor in figure 3-(c). Second, both precursors are stopped by the ELMs and the toroidal mode number seen at the same frequency of the precursor becomes negative and large in absolute value. Large phase jumps are measured during the ELMs that may affect the final results, as it will be discussed below. These time localized variations in the value of $n$ cannot be measured with standard Fourier analysis and constitute a significant step forward that has emerged from the wavelet analysis in 

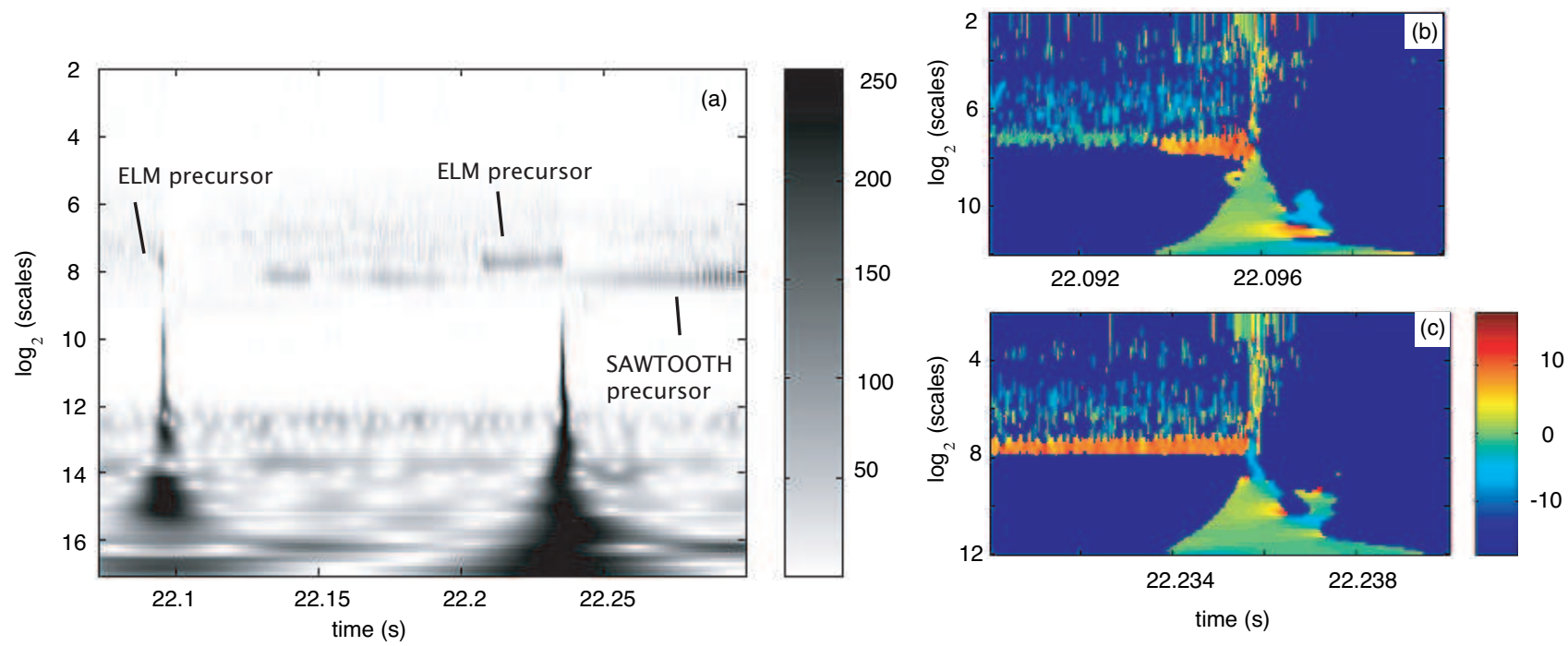

Figure 3. (Colour)(a) Wavelet scalogram. For each scale, the wavelet coefficients have been rescaled according to equation 8. Lines indicate the ELM precursors at frequency $20 \mathrm{kHz}$ and the sawtooth precursor at frequency $15 \mathrm{kHz}$. (b)-(c) Toroidal mode number (color coded) measured in a window centred on the first and on the second ELM. Dark blue corresponds to a rescaled power spectral amplitude below 20 in the left figure.

the study of the spectral properties of ELMs and of their precursors. Third, the toroidal mode numbers measured during the ELMs and at lower frequencies, are low in absolute value, namely $n=1-2$.

An excerpt of the measured magnetic perturbations in a $3 \mathrm{~ms}$ time window centred on the time at which the first ELM occurs is shown in figure 2-(c). The magnetic precursor can be seen in the time trace from the Mirnov coil, it appears approximately 2 ms before the ELM and it is not detected after the ELM. The slow asymmetric oscillation in the magnetic signal during the ELM can be captured by the Morlet wavelet coefficients in the frequency range of $[2,10] \mathrm{kHz}$, as shown in figure 2-(d). Nevertheless, as also inferred from the time trace of magnetic perturbations and from the wavelet scalogram, components at low frequency and (at relatively smaller amplitude) at high frequency, both contribute to the total perturbations measured during the ELM.

The amplitude of the spectral components in the frequency range of $[18,20] \mathrm{kHz}$, shown in figure 2-(e), increases linearly during $1.5 \mathrm{~ms}$ and then it reaches saturation, staying constant until the ELM occurs; a further increase in the amplitude is measured in this frequency range during the ELM. A similar amplitude saturation, occurring $1 \mathrm{~ms}$ after the appearance of the precursor, was observed (using Fourier techniques) on JET for precursors with longer lifetime [5].

To isolate the precursor, and to follow the time evolution of the associated toroidal mode number, we now average the cross-power spectrum over time windows of width decreasing from $1 \mathrm{~ms}$ to $10 \mu \mathrm{s}$. We fit then the wavelet cross-power spectrum in each window with a recursive fitting procedure, assuming that the peaks have a Gaussian 

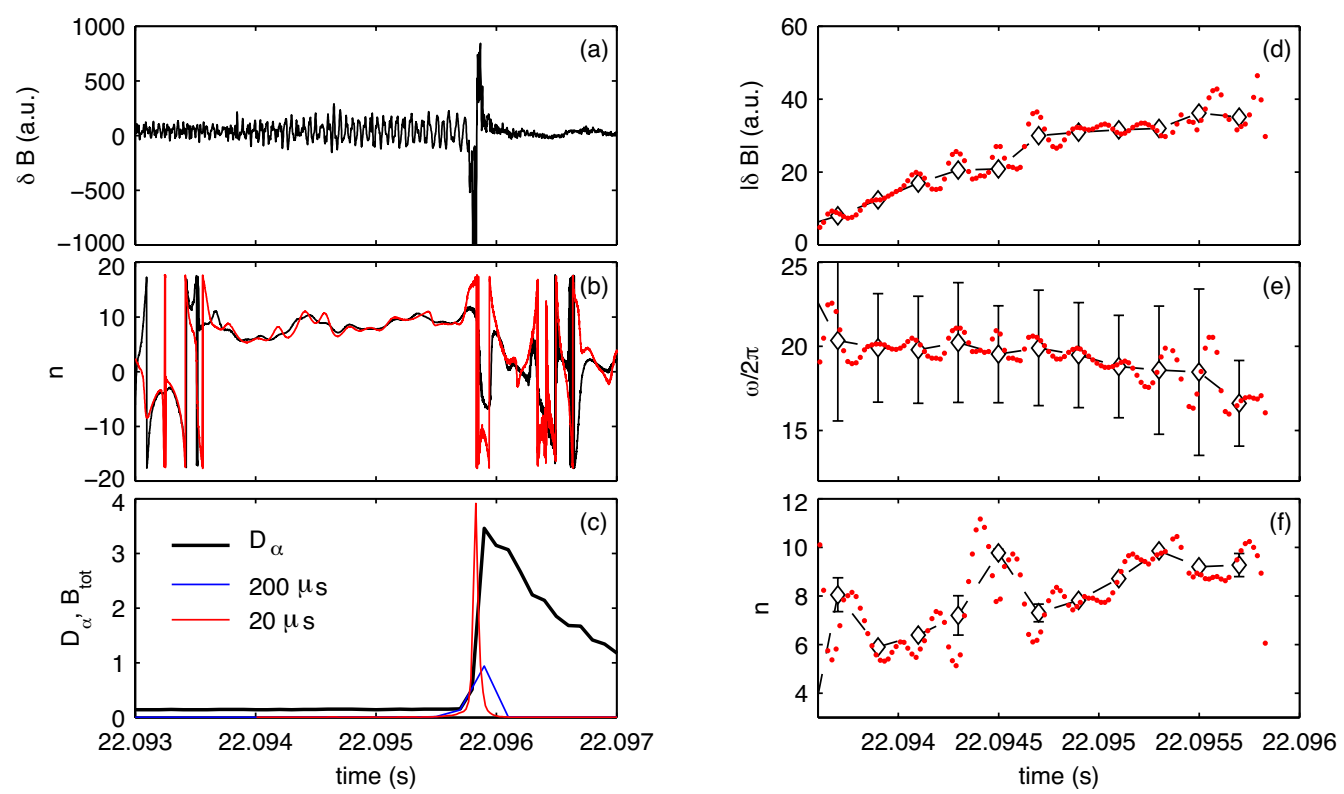

Figure 4. (Colour)(a) Time trace of the magnetic perturbations during the first ELM, also shown in figure 2-(c). (b) Toroidal mode number, extracted from the phase shift between magnetic perturbations measured by two edge Mirnov pickup coils, positioned at $R=3.881 \mathrm{~m}, z \simeq 1.01 \mathrm{~m}$ above the midplane, and toroidally separated by $\Delta \varphi=10.17$ degrees. The two curves refer to the phase shift measured at $18 \mathrm{kHz}$ (black line) and at $20 \mathrm{kHz}$ (red line). (c) Time trace of the $\mathrm{D}_{\alpha}$ emission measured in the divertor and total bispectrum $B_{\text {tot }}$, calculated averaging over $20 \mu \mathrm{s}$ and $200 \mu \mathrm{s}$. (d) Amplitude of the precursor, calculated averaging over $\tau_{2}=200 \mu \mathrm{s}(\diamond)$ and over $\tau_{1}=20 \mu \mathrm{s}(\bullet)$. (e) Same as (d), but for the averaged frequency. The vertical bars indicate the width of the peak, resulting from a Gaussian fit of the peak. (f) Same as (d) but for the averaged toroidal mode number.

envelope, according to the shape of the Morlet wavelet. The amplitude, the central frequency and the spectral width of each peak are given by the parameters of the Gaussian curve. The fitting procedure allows one to isolate peaks with amplitude significantly larger than a selected fraction of the background level. The average over windows with progressively decreasing width is a check for convergence of the calculated linear quantities, namely the amplitude and the toroidal mode number.

We will show results for averaging over two time windows, $\tau_{1}=20 \mu \mathrm{s}$, and $\tau_{2}=200 \mu \mathrm{s}$, corresponding respectively to 20 and 200 samples. The shorter interval $\tau_{1}$ is at the limit of time resolution available, since it is shorter than the wave period of the precursor, that is $50 \mu \mathrm{s}$. Averaging over $\tau_{1}$ introduces an apparent time oscillation in the amplitude, as shown in figure 4-(d), which is not present for averaging over the longer time window $\tau_{2}$. These results can be used as a check on our results observed for window $\tau_{1}$.

Figure 4 shows the time evolution of the amplitude, frequency and toroidal mode number of the first ELM precursor, starting from the time when it is detected as a coherent mode, until the ELM crash. The amplitude of the precursor increases roughly linearly (rather than exponentially) in time, although with a larger growth rate during the first $1.5 \mathrm{~ms}$. If 
we use an average over time windows shorter than those associated with the wave period, as in the case of results for $\tau_{1}$, the resultant amplitude oscillates; this artifact is a result of the spectral analysis. Generally speaking, time windows a few times longer than the wave period should be taken when calculating average linear spectral quantities, such as the amplitude and the phase shift. On JET, precursors to type-I ELMs are observed in plasmas heated by Neutral Beam Injection, at frequencies typically lower than 40 $\mathrm{kHz}$. Depending on the frequency of the precursors, variations in time of the amplitude, frequency and mode number of precursors can be followed with good statistical accuracy over time scales down to $25 \mu \mathrm{s}$.

Oscillations are also seen in the time evolution of the frequency of the precursor, which are correlated in time with the time variations of the amplitude, suggesting that this is also an effect of the averaging. These oscillations are nevertheless smaller than the spectral width of the peak; the latter is shown in figure 4-(e) as vertical bars in the results for $\tau_{2}$. The frequency of the precursor decreases from $20 \mathrm{kHz}$ to $18 \mathrm{kHz}$ before the ELM, although the total variation is comparable to the width of the peak and thus it is not significant.

The toroidal mode number is measured from the phase shift in a frequency range centred on the frequency of the mode and of width equal to the spectral width of the mode. The error in the measurement of the phase is given by the maximum dispersion associated with the average value in each time window. Variations of the toroidal mode number before the ELM are outside the range of the errors introduced from the measurement of the phase, although we cannot exclude that the small oscillations measured in the case of averaging over $\tau_{1}$ are due to the choice of the window. Importantly, the increase in the toroidal number measured at approximately $22.945 \mathrm{~s}$ is also seen in the results for the window $\tau_{2}$ and in the time evolution of the phase shift between the filtered signals, shown in figure 4 -(b); it can be therefore interpreted as a real feature in the data. A large, smooth variation in the toroidal mode number is detected approximately $150 \mu \mathrm{s}$ before the ELM burst, with the value of $n$ decreasing from approximately 9 to 4 . This is most probably due to the high level of nonlinear interactions that occur during the phase that precede the ELM crash, as shown in figure 4-(c).

The possible effect on our analysis of phase jumps of $2 \pi$ in the variation of the mode number can also be excluded. As shown in figure 4-(b), in fact, phase jumps of $2 \pi$ are measured approaching the ELM only for non-coherent spectral components, while smooth variations in the phase are associated with coherent spectral components, as is the case for the component at $18 \mathrm{kHz}$, which we plot in figure $4(\mathrm{e})$.

We have computed the wavelet bispectrum integrating over time windows of decreasing length from $1 \mathrm{~ms}$ to $20 \mu \mathrm{s}$ and, from this, the associated total bispectrum $B_{\text {tot }}$ as [9]:

$$
B_{\text {tot }}=\sum_{\omega} B_{\text {sum }}(\omega) \quad \text { with } \quad B_{\text {sum }}(\omega)=\sum_{\omega_{1}, \omega_{2}}\left|B\left(\omega_{1}, \omega_{2}\right)\right|
$$

where, for the sake of simplicity, we have written the bispectrum as a function of the frequency. The summed bispectrum $B_{\text {sum }}$ represents the contribution of nonlinear interactions at each frequency $\omega$ that are due to phase coupling of all those spectral 
components with frequency $\omega_{1}$ and $\omega_{2}$ that satisfy the resonance condition $\omega_{1}+\omega_{2}=\omega$. The total bispectrum, $B_{\text {tot }}$ quantifies the total degree of nonlinearity in the time window where the bispectrum has been computed. The total bicoherence is defined in the same way, with $\left|B\left(\omega_{1}, \omega_{2}\right)\right|$ replaced by $b^{2}\left(\omega_{1}, \omega_{2}\right)$ in (9). As discussed in [9] the total bicoherence (and the total bispectrum), calculated over an intermittent time series, give an indication of times where nonlinear interactions due to large amplitude, coherent events, are largest.

Figure 4-(c) shows the total bispectrum over plotted on the $\mathrm{D}_{\alpha}$ emission for the windows $\tau_{1}$ and $\tau_{2}$. The value of $B_{\text {tot }}$ peaks during the phase of fast rise in the $\mathrm{D}_{\alpha}$ emission, suggesting the presence of nonlinear interactions, highly localized in time, during this phase. In the case of the results for $\tau_{1}$, the value of $B_{\text {tot }}$ is larger and peaked in a shorter interval in time during the fast rise in the $\mathrm{D}_{\alpha}$. When integrating over longer time windows, as in the case of $\tau_{2}$, nonlinear interactions that are localized in time, are spread over the longer time window, resulting in a lower amplitude of $B_{\text {tot }}$. On the other hand, the smaller the integration time, the larger the error in the estimate of the bispectrum and of the bicoherence from equations (5)-(6), due to poor statistics [7].

In order to find the minimum number of samples $M$ necessary to guarantee convergence of the bispectrum and of the bicoherence, we have proceeded as follows. From the complex wavelet coefficients $W_{m}(s)$ we construct surrogate series in the frequency domain, by retaining the amplitude and randomizing the phases [11]. The resulting series have the same power spectrum as the original, as the amplitude of perturbations are unaltered, but any phase coupling is destroyed. We then calculate the bispectrum and the bicoherence of the surrogate series and compute the total bispectrum $B_{\text {tot }}$ and the total bicoherence $b_{\text {tot }}^{2}$ from (9). The value of the bispectrum and of the bicoherence associated with the surrogate series can be regarded as an estimate of the noise introduced in the computation of $B\left(\omega_{1}, \omega_{2}\right)$. Only values above this noise level should be retained as an indication of the presence of phase coupling in the time series. Whenever nonlinearities are present in the signal, the ratio between $B_{\text {tot }}$ (and $b_{\text {tot }}^{2}$ ) calculated from the original series and that calculated from the surrogate series significantly differ from unity. This ratio nevertheless depends on the number of samples used for the computation of $B\left(\omega_{1}, \omega_{2}\right)$ and we expect that it approaches unity with decreasing $M$ because of the poor statistics in the computation of the bispectrum. Conversely, if nonlinearities are absent, we expect this ratio to be close to unity, although deviations from this value can be expected for low $M$, again due to poor statistics.

The results from the original series and from the surrogate series are shown in figure 5 -(a). We can see that the ratio of the total bicoherence $b_{\text {tot }}^{2}$ of the original series to that of the surrogate series decreases from approximately 8 to 3 when the window length is reduced from $1 \mathrm{~ms}$ to $20 \mu \mathrm{s}$. Comparable values are measured also for the ratio of the total bispectrum of the original series to that of the surrogate series. For times of integration longer than $\tau_{2}$, we find that the signal-to-noise ratio is barely improved by increasing the number of samples (i.e. the length of the window). We conclude that $M=200$ is a sufficiently large number of samples to guarantee convergence of the 

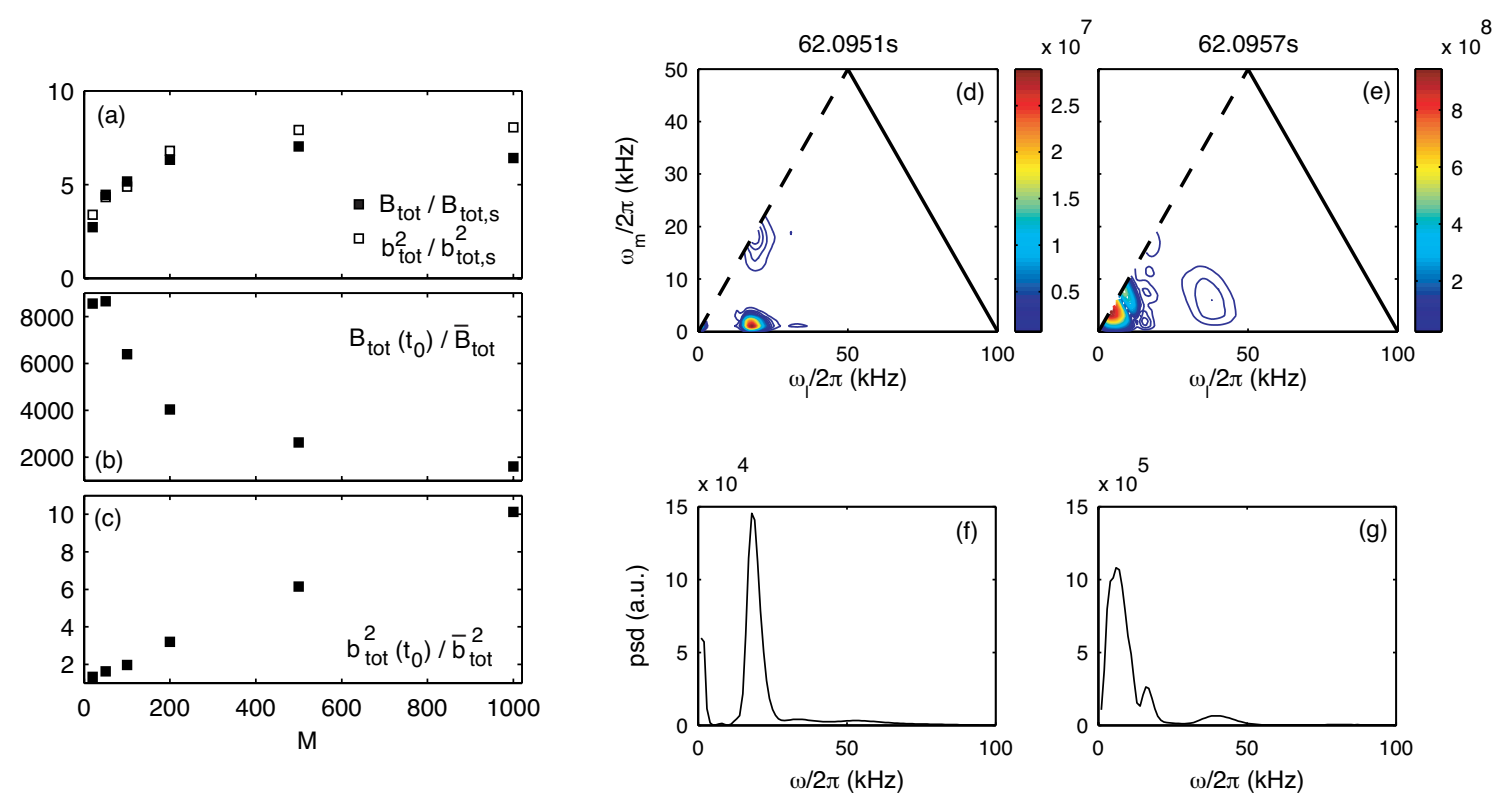

Figure 5. (Colour) Left: effect of the number of samples on the calculation of higher order spectra. (a) ratio of the total bicoherence $b_{t o t}^{2}(\square)$ and of the total bispectrum $B_{t o t}(\boldsymbol{\square})$ to the corresponding quantities, $b_{t o t, s}^{2}$ and $B_{t o t, s}$ calculated over the surrogate series. (b) ratio of the total bispectrum $B_{\text {tot }}$ computed at the time $t_{0}$ where it is maximum to the value $\bar{B}_{\text {tot }}$ computed in a $4 \mathrm{~ms}$ time window before $t_{0}$. (c) same as (b) but for the total bicoherence $b_{\text {tot }}^{2}$. Right-top: Bispectrum of the magnetic perturbations, calculated over 200 samples, measured approximately $800 \mu \mathrm{s}$ (d) and $200 \mu \mathrm{s}$ (e) before the ELM. Right-bottom: power spectral density (psd) of magnetic perturbations, arbitrary units, averaged over 200 samples, computed $800 \mu \mathrm{s}$ (f) and $200 \mu \mathrm{s}$ (g) before the ELM.

bispectrum and, thus, of the bicoherence.

Figure 5-(b) shows the ratio of $B_{\text {tot }}$ measured at the time $t_{0}$ where it is maximum, to that measured in a $4 \mathrm{~ms}$ time window before the ELM, when nonlinear interactions are negligible with respect to those occurring at $t_{0}$. The corresponding ratio for $b_{\text {tot }}^{2}$, computed at the same times, is shown in figure 5-(c). We can see that, in the case of the bispectrum, the ratio increases with decreasing the number of samples. This is due to the high localization in time of nonlinear interactions, as discussed above and also shown by the time evolution of $B_{\text {tot }}$ in figure $4-(\mathrm{c})$. Conversely, the ratio of $b_{\text {tot }}^{2}$ measured at $t_{0}$ to that measured far from $t_{0}$ increases with increasing the number of samples. This is due to the fact that the bicoherence is a biased quantity, with bias $4 \sqrt{3} / M$ and variance $4 b^{2}\left(\omega_{1}, \omega_{2}\right)\left[1-b^{2}\left(\omega_{1}, \omega_{2}\right)\right] / M[12]$. Both the bias and the variance decrease with increasing the number of samples. We find that, when we decrease the time window length down to values comparable to the wave period, the level of total bicoherence increases in all time windows and there is no clear separation between the value of $b_{\text {tot }}^{2}$ measured at $t_{0}$ and that measured away from it. For time windows comparable to the wave period, the value of $b_{\text {tot }}^{2}$ is not a good indication of the localization in time of nonlinear interactions, as indicated by the low values in figure 5-(c), the total bispectrum 
should be used instead.

Since nonlinear interactions during the ELMs are highly localized in time during the fast rise in the $\mathrm{D}_{\alpha}$ emission, which occurs on JET typically over time scales comparable to $\tau_{2}$, shorter times of integration are needed to follow the nonlinear dynamics of ELMs during this phase. Nevertheless, as shown in figure 5-(c), the number of samples should be taken as large as possible to guarantee convergence of the bicoherence. In those cases where a large number of ELMs with similar characteristics are present in the same discharge, such as in the case of type-III ELMs, or in the case of regular type-I ELMs, the signal-to-noise ratio can be increased calculated higher order spectra over ensembles of ELMs.

Figures 5(d)-(e) show the bispectrum calculated by averaging over $\tau_{2}$, for (d) $600 \mu$ s and (e) $200 \mu \mathrm{s}$ before $t_{0}$. As shown in the figure, before the ELM and during the phase where we have seen that the amplitude of the precursors increases linearly, the bispectrum is large for interactions involving the precursor (at $\omega / 2 \pi \sim 20 \mathrm{kHz}$ ) and spectral components with the lowest frequencies, namely $\omega / 2 \pi<5 \mathrm{kHz}$. In correspondence of the generation of the second harmonics, for $\left(\omega_{1} / 2 \pi, \omega_{2} / 2 \pi\right)=(20,20)$, the amplitude of the bispectrum is above the background level, although it is approximately $20 \%$ of the peak value. The phase coupling among low frequency spectral components increases as we approach $t_{0}$, as shown in figure $5(\mathrm{e})$. In general, the range of frequencies involved in the interactions and their strength depend on the details of the experiment and on the global spectral features. For example, when other modes are present in the spectrum, such as washboard modes [13], phase coupling between these modes and the precursor may be measured [5].

\subsection{Type-III ELM precursors and post-cursors}

In order to underline the advantage of the Morlet wavelet analysis for identifying short-lived precursor and post-cursors, we show in figure 6 an example of the wavelet spectrogram computed in the case of a H-mode plasma with type-III ELMS on JET (pulse \#52308). Interestingly, wavelet analysis reveals in this case features that cannot be captured with standard Fourier analysis. Both pre-cursors and post-cursors to type-III ELMs are detected in the wavelet spectrogram. Precursors with lifetime typically below $5 \mathrm{~ms}$ are visible at scales $\log _{2}(s) \sim 7$, corresponding to frequencies of approximately $65 \mathrm{kHz}$. They have toroidal mode numbers $n \sim 10$. A post-cursor with lifetime of approximately $5 \mathrm{~ms}$ is detected at $t \sim 22.63 \mathrm{~s}$. Harmonics of the post-cursor are visible, whose frequency decreases with time and whose toroidal mode numbers start from $n=1$ at the lowest frequency harmonics and increase with steps of $\Delta n=1$ for higher harmonics. Post-cursors with lifetime below $1 \mathrm{~ms}$ are detected in

figure 6 occurring right after other ELMs, for example at $22.43 \mathrm{~s}$ and at $22.5 \mathrm{~s}$. They are not visible in the Fourier spectrogram, but can be identified by the Morlet wavelet. 


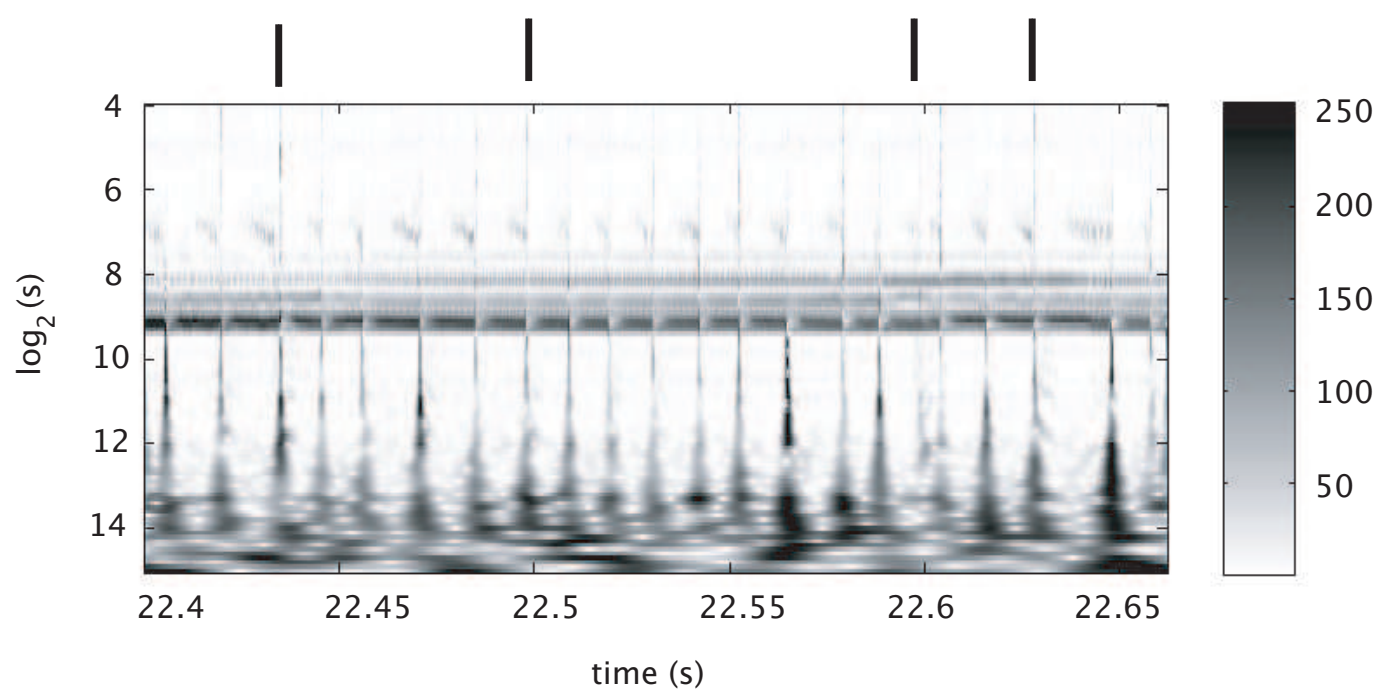

Figure 6. JET pulse \#52308. Wavelet spectrogram computed from (3), taking $\omega_{0}=3 \pi$ in 1 for a better frequency localization. Lines above the figure point to some of the post-cursors.

\section{Conclusions}

By analysing a time series that has been previously treated with Fourier spectral analysis, we demonstrate that wavelet analysis is suitable to identify spectral features of ELM precursors, such as the toroidal mode number and the nonlinear evolution over time scales comparable with the wave period. The calculated toroidal mode numbers are consistent with the results from the Fourier analysis in the case of precursors with lifetime longer than a few ms. In the case of precursors with lifetime shorter than $1 \mathrm{~ms}$, where Fourier analysis cannot capture the spectral features, wavelet analysis is reliable to extract the toroidal mode number with good statistics in time. Importantly, variations of the amplitude, frequency and toroidal mode number of precursors can be followed over time scales comparable with the wave period. It is shown that, using Morlet wavelets, coherent modes in the power spectrum, such as precursors and post-cursors to typeIII ELMs, are detected over time windows comparable to a few times the acquisition time. The positive results of wavelet analysis applied to precursors encourage their application to the study of the spectral features of ELMs themselves, including their toroidal mode number, important for e.g. assessing the linear stability with finite ion Larmor radius effects included. Preliminary results from the computation of spectra in ELMy H-mode plasmas with wavelets indicate that the toroidal mode number of type-I ELMs is different from the toroidal mode number of precursors, but it may correlate with that of post-cursors [14]. An extended comparison of the spectral features of both type-I and type-III ELMs, including the correlation with the toroidal mode numbers of precursors and post-cursors is work in progress and will constitute the subject of a 
separate work.

\section{Acknowledgments}

This work has been conducted under the European Fusion Development Agreement. The views and opinions expressed herein do not necessarily reflect those of the European Commission. We acknowledge the UK EPSRC for support.

\section{References}

[1] Zohm M, Plasma Phys. Control. Fusion, 38, 105 (1996).

[2] Connor J. W, Plasma Phys. Control. Fusion, 40, 191 (1998).

[3] Connor J. W, Plasma Phys. Control. Fusion, 40, 531 (1998).

[4] Kamiya K, Asakura N, Boedo J, et. al, Plasma Phys. Control. Fusion, 49, S43 (2007).

[5] Perez C. P, Koslowski H. R, Huysmans G. T. A, et al, Nucl. Fusion, 44, 609 (2004).

[6] Mallat S, A wavelet tour of signal processing, chapter 4. Academic Press, Cambridge, (2001).

[7] Kim Y. C and Powers E. J, Phys. Fluids, 21, 1452 (1978).

[8] Kim Y. C and Powers E. J, IEEE trans. Plasma sci., PS-7, 120 (1979).

[9] van Milligen B. Ph, Hidalgo C, and Sánchez E. Phys. Rev. Lett., 74, 395 (1995).

[10] Heeter R F, Fasoli A F, Ali-Arshad S and Moret J M, Rev. Sci, Instrum., 71, 4092 (2000).

[11] Theiler J, Eubank S, Longlin A, et al, Physica D, 58, 77 (1992).

[12] Hinich M J and Clay C S, Rev. Geoph., 6, 347 (1968).

[13] Smeulders P, Conway G D, Alper et al, Plasma Phys. Control. Fusion, 41, 1303 (1992).

[14] Poli FM, Sharapov SE et al, Proceedings of the $35^{\text {th }}$ EPS Plasma Physics Conference, Hersonissos, Crete, Greece, (2008). 